\title{
Anxiety and hypertension in the COVID-19 era: how is the central autonomic network linked?
}

\author{
Michiaki Nagai $^{1} \cdot$ Masaya Kato $^{1} \cdot$ Dote Keigo $^{1}$
}

Received: 11 January 2022 / Accepted: 18 January 2022 / Published online: 18 February 2022

(c) The Author(s), under exclusive licence to The Japanese Society of Hypertension 2022

Fear and avoidance of trigger cues are common to many anxiety disorders and resemble the arousal and avoidance responses shown to conditioned fear cues [1]. Thus, a common element of anxiety disorders might be an abnormally elevated fear response. Coronavirus disease 2019 (COVID-19) is caused by severe acute respiratory syndrome coronavirus 2 (SARS-CoV-2) and is currently a worldwide pandemic. In the general population during the COVID-19 epidemic, up to half of UK adults were reported to have increased anxiety, depression, or stress due to fear of contracting SARS-CoV-2 [2]. Concomitant with increased anxiety, poor sleep hygiene has also been highlighted during the COVID-19 pandemic [3].

The paper by Zhang et al. [4]. in this issue of the Journal provides several new insights into the relationship between anxiety and elevated blood pressure (BP) in the COVID-19 era. Hypertensive patients with an increased level of anxiety showed higher morning systolic blood pressure (SBP) than those without anxiety. In addition, significant betweengroup differences were observed in SBP changes during the outbreak and plateau periods. During the 1-year follow-up period, patients with anxiety had a significantly increased risk of cardiovascular events [4].

It has been speculated that a central autonomic network (CAN) comprising the peri-aqueductal gray matter, parabrachial nucleus, nucleus tractus solitarius, ventrolateral medulla, hypothalamus, amygdala, and insular cortex (Ic) regulates the human cardiovascular system and that the network of these cortical regions is necessary to regulate the CAN in response to emotional stress [5, 6]. The CAN functions as an integral component of an internal regulation system with which the brain controls the visceromotor and

Michiaki Nagai

nagai10m@r6.dion.ne.jp

1 Department of Cardiology, Hiroshima City Asa Hospital, Hiroshima, Japan neuroendocrine systems [5]. The involvement of a network within the CAN consisting of the amygdala and Ic was shown to be associated with the relationship between the processing of emotional information and autonomic nervous system responses [7]. Specifically, in a meta-analysis of functional neuroimaging studies of anxiety, hyperactivation in the amygdala and Ic was more frequently observed in social anxiety disorder and specific phobia [8] (Fig. 1).

The amygdala forms the core of a neural system for processing fearful and threatening stimuli, and acute emotional stressors have been suggested to induce amygdala activation in hypertension [9]. Several neuroimaging studies showed that activation of the Ic is also involved in the processing of negative emotions such as anger, fear, and anxiety [7]. When an individual confronts a danger to fearful things, a combination of autonomic, behavioral, and cognitive responses occurs for self-preservation. These features are shown to be merged at the Ic, which fine-tunes behavioral responses to match the level of expected danger for fearful things [10].

In spontaneously hypertensive rats, increased sympathetic nervous system (SNS) activity was shown to be attributed to alterations in the neurovascular unit in the Ic, leading to changes in neurogenic BP elevation [11]. In contrast, Ic was reported to store immune-related information using activitydependent cell labeling in mice [12]. Clinical studies revealed that the damage involving the Ic is associated with a nocturnal BP rise, cardiac overload, a higher plasma level of catecholamine, and a poor prognosis [5].

In the Jichi Medical School ABPM Study Wave 2 Core, left Ic atrophy had a significant correlation with the level of brain natriuretic peptide, and right Ic atrophy had a significant correlation with the level of noradrenaline [5]. In addition, Ic atrophy was associated with disrupted diurnal ambulatory BP rhythm [13]. Patients who had an ischemic stroke involving the Ic more frequently showed a rise in nocturnal BP, and they presented with higher norepinephrine levels than patients without Ic-involved ischemia [14]. Thus, in elderly hypertensive patients during the COVID-19 era, disruption of CAN, including Ic, might 


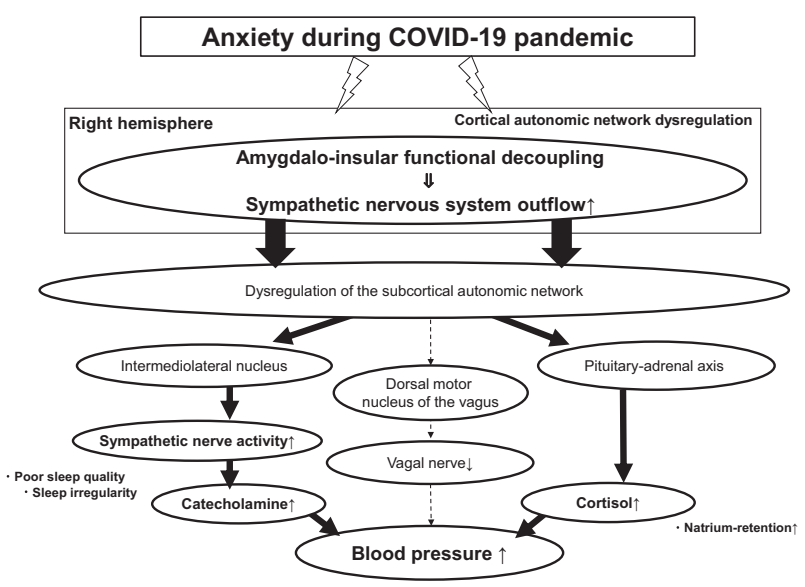

Fig. 1 A possible pathway for the relationship between anxiety and elevated blood pressure in the COVID-19 era. COVID-19 indicates coronavirus disease 2019

cause the predominant sympathovagal balance to increase SNS activity, leading to higher morning BP, as reported by Zhang et al. [4].

A study using experimental stroke models reported that occlusion of the right middle cerebral artery resulted in neurochemical derangements in the ipsilateral Ic and amygdala, which led to an enhancement of sympathetic outflow to the cardiovascular system, resulting in an increase in synaptic norepinephrine levels [15]. Enhanced sympathetic outflow could also occur as a result of emotional stimulation, such as anxiety, which might induce cardiovascular system changes similar to those observed in acute cerebrovascular disease [5].

Close reciprocal anatomical functional connections between the Ic and amygdala have been shown [15]. A neuropeptide $\mathrm{Y}$ increase in the basolateral nucleus of the amygdala as well as leucine-enkephalin, dynorphin, and neurotensin increases in the central nucleus of the amygdala were seen only when the ipsilateral Ic was lesioned [15]. The axonal transport of signals from the Ic to the amygdala was critical in mediating the neuropeptide changes in the amygdala seen after stroke [15]. Thus, the functional disrupted coupling of the amygdalo-insular connection would be an important consideration when investigating the pathophysiology of anxiety-related BP elevation (Fig. 1).

Until now, there have been few reports assessing the relationships of anxiety with elevated BP and unfavorable outcomes in the COVID-19 era. In addition to strict BP control, it might be important to minimize anxiety to prevent further cardiovascular events. The data presented in the study by Zhang et al. [4]. thus make an important contribution, provided that they are considered within the context of the precise pathophysiology underlying the relationship between anxiety and elevated $\mathrm{BP}$ in the COVID-19 era.

\section{Compliance with ethical standards}

Conflict of interest The authors declare no competing interests.

Publisher's note Springer Nature remains neutral with regard to jurisdictional claims in published maps and institutional affiliations.

\section{References}

1. Grillon C. Startle reactivity and anxiety disorders: aversive conditioning, context, and neurobiology. Biol Psychiatry. 2002;52:958-75.

2. Duffy B. Life under lockdown: Coronavirus in the UK. Kings College London: The Policy Institute; 2020.

3. Anwer S, Li H, Antwi-Afari MF, Shaphe MA, Alghadir A, Wong AYL. Evaluation of sleep habits, generalized anxiety, perceived stress, and research outputs among postgraduate research students in Hong Kong during the coronavirus (COVID-19) pandemic. J Multidiscip Health. 2021;14:3135-49.

4. Zhang S, Zhong Y, Wang L, Yin X, Li Y, Liu Y, et al. Anxiety, home blood pressure monitoring, and cardiovascular events among older hypertension patients during COVID-19 pandemic. Hypertens Res. 2022 https://doi.org/10.1038/s41440-022-00852-0.

5. Nagai M, Dote K, Kato M, Sasaki S, Oda N, Kagawa E, et al. (2017). The insular cortex and Takotsubo cardiomyopathy. Curr Pharm Des. 2017;23:879-88.

6. Goldstein DS. The extended autonomic system, dyshomeostasis, and COVID-19. Clin Auton Res. 2020;30:299-315.

7. Nagai M, Kishi K, Kato S. Insular cortex and neuropsychiatric disorders: a review of recent literature. Eur Psychiatry. 2007;22:387-94.

8. Etkin A, Wager TD. Functional neuroimaging of anxiety: a metaanalysis of emotional processing in PTSD, social anxiety disorder, and specific phobia. Am J Psychiatry. 2007;164:1476-88.

9. Sheng ZF, Zhang H, Zheng P, Chen S, Gu Z, Zhou JJ, et al. Impaired Kv7 channel activity in the central amygdala contributes to elevated sympathetic outflow in hypertension. Cardiovasc Res. 2022;118:585-96.

10. Klein AS, Dolensek N, Weiand C, Gogolla N. Fear balance is maintained by bodily feedback to the insular cortex in mice. Science. 2021;374:1010-5.

11. Marins FR, Iddings JA, Fontes MA, Filosa JA. Evidence that remodeling of insular cortex neurovascular unit contributes to hypertension-related sympathoexcitation. Physiol Rep. 2017;5: e13156.

12. Koren T, Yifa R, Amer M, Krot M, Boshnak N, Ben-Shaanan TL, et al. Insular cortex neurons encode and retrieve specific immune responses. Cell. 2021;184:5902-15.

13. Nagai M, Hoshide S, Ishikawa J, Shimada K, Kario K. Insular cortex atrophy as an independent determinant of disrupted diurnal rhythm of ambulatory blood pressure in elderly hypertension. Am J Hypertens. 2009;22:723-9.

14. Sander D, Klingelhofer J. Changes of circadian blood pressure patterns after hemodynamic and thromboembolic brain infarction. Stroke. 1994;25:1730-7.

15. Cheung RT, Cechetto DF. Neuropeptide changes following excitotoxic lesion of the insular cortex in rats. J Comp Neurol. 1995;362:535-50. 